\title{
Building Virtual Earth Observatories using Ontologies and Linked Geospatial Data*
}

\author{
Manolis Koubarakis \\ Dept. of Informatics and Telecommunications \\ National and Kapodistrian University of Athens \\ Athens, Greece \\ koubarak@di.uoa.gr
}

\section{Introduction}

Advances in remote sensing technologies have enabled public and commercial organizations to send an ever-increasing number of satellites in orbit around Earth. As a result, Earth Observation (EO) data has been constantly increasing in volume in the last few years, and it is currently reaching petabytes in many satellite archives. For example, the multi-mission data archive of the TELEIOS partner German Aerospace Center (DLR) is expected to reach $2 \mathrm{~PB}$ next year, while ESA estimates that it will be archiving $20 \mathrm{~PB}$ of data before the year 2020. As the volume of data in satellite archives has been increasing, so have the scientific and commercial applications of EO data. Nevertheless, it is estimated that up to $95 \%$ of the data present in existing archives has never been accessed, so the potential for increasing exploitation is very big.

TELEIOS ${ }^{1}$ is a recent European project that addresses the need for scalable access to PBs of Earth Observation data and the effective discovery of knowledge hidden in them. TELEIOS started on September 2010 and it will last for 3 years. In the first one and a half years of the project, we have made significant progress in the development of state-of-the-art techniques in Scientific Databases, Semantic Web and Image Mining and have applied them to the management of EO data.

In the rest of this technical communication we outline the contributions of TELEIOS, and explain why it goes significantly beyond operational systems currently deployed in various EO data centers and Earth Observation portals such as EOWEB-NG. We also present its main technical contributions related to ontologies and linked geospatial data.

\section{Basic Concepts of the TELEIOS Earth Observatory}

Satellite missions continuously send to Earth huge amounts of EO data providing snapshots of the surface of the Earth or its atmosphere. The management of

\footnotetext{
* This work has been funded by the FP7 project TELEIOS (257662).

${ }^{1}$ http://www. earthobservatory.eu/
} 


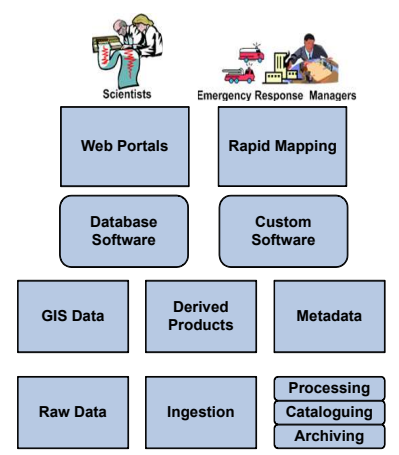

(a) Concept view of a state-of-the-art EO data center

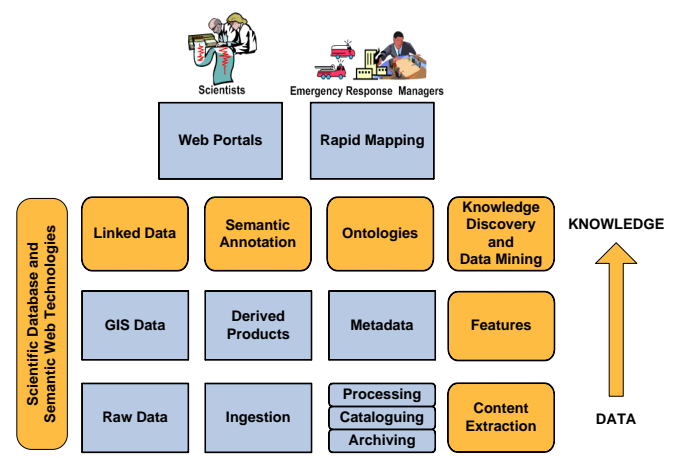

(b) Concept view of the TELEIOS Earth Observatory

Fig. 1. Pre-TELEIOS EO data centers and the TELEIOS Earth Observatory

the so-called payload data is an important activity of the ground segments of satellite missions. Figure 1(a) gives a high-level view of some of the basic data processing and user services available at EO data centers today, e.g., at the German Remote Sensing Data Center (DFD) of TELEIOS partner DLR through its Data Information and Management System (DIMS) [8].

Raw data, often from multiple satellite missions, is ingested, processed, cataloged and archived. Processing results in the creation of various standard products (Level 1, 2, etc., in EO jargon; raw data is Level 0) together with extensive metadata describing them. For example, in the NOA application of TELEIOS [4], images from the SEVIRI sensor are processed (cropped, georeferenced and run through a pixel classification algorithm) to detect pixels that are hotspots. Then these pixels are stored as standard products in the form of shapefiles. Raw data and derived products are complemented by auxiliary data, e.g., various kinds of geospatial data such as maps, land use/land cover data, etc.

Raw data, derived products, metadata and auxiliary data are stored in various storage systems and are made available using a variety of policies depending on their volume and expected future use. For example, in the TerraSAR-X archive managed by DFD, long term archiving is done using a hierarchy of storage systems (including a robotic tape library) which offers batch to near-line access, while product metadata are available on-line by utilizing a relational DBMS and an object-based query language [8].

EO data centers such as DFD also offer a variety of user services. For example, for scientists that want to utilize EO data in their research, DFD offers the Web interface EOWEB-NG ${ }^{2}$ for searching, inspection and ordering of products. Space agencies such as DLR and NOA might also make various other services available aimed at specific classes of users. For example, the Center for Satellite Based

\footnotetext{
2 https://centaurus.caf.dlr.de:8443/
} 
Crisis Information (ZKI) ${ }^{3}$ of DLR provides a $24 / 7$ service for the rapid provision, processing and analysis of satellite imagery during natural and environmental disasters, for humanitarian relief activities and civil security issues worldwide. Similar emergency support services for fire mapping and damage assessment are offered by NOA through its participation in the GMES SAFER program.

Let us now summarize the TELEIOS advancements to today's state of the art in EO data processing that are shown graphically with yellow color in Figure $1(b)$.

Firstly, traditional raw data processing is augmented by content extraction methods that deal with the specificities of satellite images and derive image descriptors (e.g., texture features, spectral characteristics of the image, etc.). Knowledge discovery techniques combine image descriptors, image metadata and auxiliary data (e.g., GIS data) to determine concepts from a domain ontology (e.g., forest, lake, fire, burned area, etc.) that characterize the content of an image [2].

Hierarchies of domain concepts are formalized using OWL ontologies and are used to annotate standard products. Annotations are expressed in RDF and are made available as linked data [1] so that they can be easily combined with other publicly available linked data sources (e.g., GeoNames, LinkedGeoData, DBpedia) to allow for the expression of rich user queries.

Web interfaces to EO data centers and specialized applications (e.g., rapid mapping) can now be improved significantly by exploiting the semanticallyenriched standard products and linked data sources made available by TELEIOS. For example, the advanced TELEIOS interface to EO data archives that is now being developed on top of the system Strabon based on stRDF and stSPARQL can enable end-users to pose very expressive queries.

stRDF is an extension of the W3C standard RDF that allows the representation of geospatial data that changes over time $[5,6]$. stRDF is accompanied by stSPARQL, an extension of the query language SPARQL 1.1 for querying and updating stRDF data. stRDF and stSPARQL use OGC standards (Well-Known Text and Geography Markup Language) for the representation of temporal and geospatial data $[3,6]$.

In TELEIOS, stRDF is used to represent satellite image metadata (e.g., time of acquisition, geographical coverage), knowledge extracted from satellite images (e.g., a certain image pixel is a fire hotspot) and auxiliary geospatial data sets encoded as linked data. One can then use stSPARQL to express in a single query an information request such as the following: "Find an image taken by a Meteosat second generation satellite on August 25, 2007 which covers the area of Peloponnese and contains hotspots corresponding to forest fires located within $2 \mathrm{~km}$ from a major archaeological site". Encoding this information request today in a typical interface to an EO data archive such as EOWEB-NG is impossible, because domain-specific concepts such as "forest fires" are not included in the archive metadata, thus they cannot be used as search criteria. In EOWEB-NG and other similar Web interfaces, search criteria include a hierarchical organiza-

\footnotetext{
${ }^{3}$ http://www.zki.dlr.de/
} 
tion of available products (e.g., high resolution optical data, Synthetic Aperture Radar data, their subcategories, etc.) together with a temporal and geographic selection menu.

We have been developing image information mining techniques that allow us to characterize satellite image regions with concepts from appropriate ontologies (e.g., landcover ontologies with concepts such as water-body, lake, forest, etc., or environmental monitoring ontologies with concepts such as forest fires, flood, etc.). These concepts are encoded in OWL ontologies and are used to annotate EO products. In this way, we attempt to close the semantic gap that exists between user requests and searchable information available explicitly in the archive.

But even if semantic information was included in the archived annotations, one would need to join it with information obtained from auxiliary data sources to answer the above query. Although such open sources of data are available to EO data centers, they are not used currently to support sophisticated ways of end-user querying in Web interfaces such as EOWEB-NG. In TELEIOS, we assume that auxiliary data sources, especially geospatial ones, are encoded in stRDF and are available as linked geospatial data, thus stSPARQL can easily be used to express information requests such as the above. The linked data web is being populated with geospatial data quickly, thus we expect that languages such as stSPARQL (and the related OGC standard query language GeoSPARQL [7]) will soon be mainstream extensions of SPARQL that can be used to access such data effectively.

\section{References}

1. Bizer, C., Heath, T., Berners-Lee, T.: Linked data-the story so far. Int. J. Semantic Web Inf. Syst. (2009)

2. Dumitru, C.O., Molina, D.E., Cui, S., Singh, J., Quartulli, M., Datcu, M.: KDD concepts and methods proposal: report \& design recommendations. Del. 3.1, TELEIOS project (2011)

3. Garbis, G., Mpereta, K., Karpathiotakis, M., Kyzirakos, K., Nikolaou, B., Sioutis, M., Vassos, S., Miliaraki, I., Papadaki, K., Koubarakis, M., Members, C.: An implementation of a temporal and spatial extension of RDF and SPARQL on top of MonetDB - phase I. Del. 4.1, TELEIOS project (2012)

4. Kontoes, H., Papoutsis, I., Michail, D.: Requirements specification for the real-time fire monitoring application. Del. D7.1, TELEIOS (2012)

5. Koubarakis, M., Kyzirakos, K.: Modeling and querying metadata in the semantic sensor web: The model stRDF and the query language stSPARQL. In: ESWC (2010)

6. Koubarakis, M., Kyzirakos, K., Nikolaou, B., Sioutis, M., Vassos, S.: A data model and query language for an extension of RDF with time and space. Del. 2.1, TELEIOS project (2011)

7. OGC, O.G.C.I.: GeoSPARQL - A geographic query language for RDF data (November 2010)

8. Wolfmüller, M., Dietrich, D., Sireteanu, E., Kiemle, S., Mikusch, E., Böttcher, M.: Data Flow and Workflow Organization - The Data Management for the TerraSAR-X Payload Ground Segment. IEEE TGRS (2009) 